Cahiers de recherches médiévales

15 | 2008

La Tentation du parodique dans la littérature médiévale

\title{
Tentations et mariage sataniques dans Richard sans
} Peur

Le détournement des modèles allégoriques et féeriques

Élisabeth Gaucher

\section{OpenEdition \\ Journals}

Édition électronique

URL : https://journals.openedition.org/crm/5593

DOI : $10.4000 / \mathrm{crm} .5593$

ISSN : $1955-2424$

Éditeur

Honoré Champion

Édition imprimée

Date de publication : 20 juin 2008

Pagination : 73-86

ISSN : 1272-9752

Référence électronique

Élisabeth Gaucher, "Tentations et mariage sataniques dans Richard sans Peur », Cahiers de recherches médiévales [En ligne], 15 | 2008, mis en ligne le 20 juin 2011, consulté le 15 décembre 2022. URL:

http://journals.openedition.org/crm/5593 ; DOI : https://doi.org/10.4000/crm.5593 


\title{
酷RM
}

\section{Tentations et mariage sataniques dans Richard sans Peur: le détournement des modèles allégoriques et féeriques}

\begin{abstract}
In its printed version of 1496 the verse romance Richard sans Peur comes as a series of fantastic adventures all of which leave the hero unmoved. One of them, reminiscent of the Livre de Baudouin de Flandre, actually deals with the marriage to the devil theme and reads as a parody of courtly and fairy love. The attitude of the protagonist who indulges in a game with his daemon seems to convey the pleasure of reading to be enjoyed in a text pervaded with «renardie».

Résumé : Conservé dans une version imprimée de 1496, le roman en vers de Richard sans Peur se présente sous la forme d'une suite d'aventures fantastiques qui laissent impassible le héros. L'une d'elles, le mariage avec le diable, que l'on trouve aussi dans le Livre de Baudouin de Flandre, donne lieu à une parodie de l'amour féerique et courtois. Mais dans l'attitude du protagoniste qui se plaît à jouer avec son démon, se devine le plaisir de la lecture, auquel invite un texte placé sous le signe de la « renardie».
\end{abstract}

L'auteur du roman de Richard sans Peur, dont la version en vers figure, avec la Vie du terrible Robert le Dyable, dans un incunable de 1496, fait appel, dès le prologue, à l'expérience littéraire de son lecteur en plaçant le récit dans un rapport d'intertextualité :

Il n'est gueres cy homme, de ce ne doubtés mye,

Qui n'aye de bout en bout toute l'istoire ouÿe

De Robert le Diable qui fut de Normendie,

Mais de Richart son filz vous vueil compter la vie. ${ }^{1}$

De l'histoire du père, né des œuvres du diable et converti à Dieu au prix d'une pénitence exemplaire, à celle du fils, occupé à chevaucher nuit et jour en quête d'un sentiment qui lui fait défaut, la peur, dont un démon fait le pari de lui inspirer le goût en lui tendant une série de pièges, l'aventure fantastique subit une dévaluation parodique : alors que Robert, dès la révélation du secret de sa naissance, considérant avec effroi et répulsion les forces maléfiques qui se disputaient son âme, mettait toute sa force à les combattre, Richard se complaît dans un jeu diabolique qui ne s'engage plus de l'intérieur mais à l'extérieur de son moi. Au terme de sept séquences qui n'ont pu ébranler son impassibilité, le héros, à son insu, engage à son service son démon, qui l'aide à repousser l'envahisseur anglais, en échange de quoi il doit le suivre en enfer : cette huitième et dernière aventure, étrangère à toute quête de peur, se solde par un duel judiciaire d'où le duc de Normandie ressort libéré de son orgueil et conscient de ses devoirs chevaleresques et chrétiens.

${ }^{1}$ Richard sans Peur, ed. from D. J. Conlon, Chapel Hill, 1977, v.5-8.

Cahiers de Recherches Médiévales, 15, 2008 
Toutes les épreuves de Richard sont régies par une puissance surnaturelle qui, dans sa prétention à maîtriser le destin du héros, dispute au clerc son « rôle d'instance poétique régulatrice $»^{2}$. Mais ces tentations diaboliques ne répondent pas seulement à un enjeu structurel et à une technique de réécriture: nous verrons qu'elles servent de support, au niveau interprétatif, à un jeu sur la tradition allégorique. L'une d'elles, le mariage, retiendra ensuite notre attention, dans ses rapports avec le roman de Baudouin de Flandre. Enfin, il s'agira d'éclairer le « beau jeu » de notre auteur qui, derrière son anonymat, renouvelle l'écriture de la « renardie ».

\section{"Résolution héroïque » et refus de l'allégorèse}

Le roman, construit à la manière d'un heptameron, s'offre comme une compilation d'aventures fantastiques sur lesquelles s'exerce la dévaluation parodique. Il s'inscrit dans la même tendance syncrétique que les romans arthuriens tardifs, où, plutôt que de remonter aux hypotextes du XII ${ }^{\mathrm{e}}$ siècle, il a bien pu trouver la matière de son inspiration.

Désireux de «querir adventure », et plus particulièrement «homme qui luy deïst riens qui luy desplairait » (v.23-24), Richard chevauche inlassablement dans sa forêt, où Brundemor se présente à lui sous la forme de diverses «semblances ». Les pièges tendus par le démon se succèdent sur le mode du continuum. Sans référer aux sources où s' origine chacune de ces « diabolies $)^{3}$, nous nous bornerons ici à en faire l'inventaire pour étudier l'accueil que leur réserve le héros.

1) Une troupe de chats-huants assaille Richard, qui conjure le danger en imitant leur cri (la parodie est déjà à l'œuvre...), sans plus d'égard pour ces oiseaux de mauvais augure.

2) Richard entend pleurer une petite fille au sommet d'un arbre. Il la délivre et la confie à ses forestiers, plus attentif à la perspective d'un futur mariage qu'au travestissement d'un démon inversant le symbolisme de l'arbre de Jessé.

3) La «mesnie Hellequin » erre sur les terres du duc de Normandie. Celui-ci, insensible au châtiment qu'endure la troupe des revenants et au danger qu'encourt tout homme qui leur adresse la parole, s'entretient de son avenir avec leur chef et reçoit en cadeau un riche drap de soie.

4) La vision d'un magnifique pommier, suivie de son étrange disparition, suscite moins le souvenir de l'arbre biblique aux fruits défendus que l'empressement à faire fructifier les pépins de trois pommes que le duc, en bon Normand, a pris soin de récolter.

\footnotetext{
${ }^{2}$ C. Ferlampin-Acher, Merveilles et topique merveilleuse dans les romans médiévaux, Paris, Champion, 2003 (N.B.M.A., 66), p. 514.

${ }^{3}$ Sur cet aspect, nous renvoyons à nos articles : « La parodie du fantastique dans Richard sans Peur », "Furent les merveilles pruvees et les aventures truvees " (Hommage à Francis Dubost), Paris, Champion, 2005 («Colloques, congrès et conférences sur le Moyen Âge », 6), p.247-261; «Richard sans Peur: le jeu avec le diable », à paraître en 2009 dans CAMAREN, 3 (Diables et diableries).
} 
5) Les turbulences d'un cadavre habité par le diable, dont Richard vient à bout par la force de son bras, se manifestent dans une chapelle abandonnée où le duc, en oubliant ses gants, semble défier plutôt que fuir son adversaire démoniaque.

6) Le conseil nocturne, où Richard est appelé à arbitrer la dispute d'un ange et d'un démon qui revendiquent l'âme d'un moine débauché, donne à voir un duc paisible, rendant justice dans sa chambre à coucher en faveur d'un pécheur auquel, loin de s'identifier, il accorde une seconde chance.

7) Célébré sept ans après sa rencontre, le mariage avec la fillette recueillie dans l'arbre met un terme à ces épreuves terrestres et n'aboutit qu'à éveiller chez Richard, dupé par le scénario de la fausse morte que joue Brundemor avant de s'enfuir, la rancœur d'un mari trompé et bafoué dans sa dignité féodale.

Ainsi, le récit de Richard sans Peur s'offre comme une anthologie d'aventures stéréotypées, ordonnées selon un schéma linéaire, paratactique. Mais si chaque séquence narrative aboutit à une clôture sur le plan structurel, ces microdénouements jouent avec l'attente du lecteur et n'apportent pas de résolution satisfaisante au niveau cognitif: la resémantisation des topoi s'opère sur le mode déceptif.

En effet, Richard ne s'étonne ni ne questionne, du moins en ce qui concerne la causalité surnaturelle des aventures. Le fantastique, qui repose sur la poétique de la peur et de l'incertain, ne peut donc plus fonctionner. Désormais, le héros sait comment réagir face à la merveille: comme le lecteur, il semble conditionné par toute une tradition de merveilleux romanesque. Bien plus, indifférent aux causes, Richard déporte son intérêt sur les effets, bénéfiques ou non : les nouvelles dispositions psychologiques du héros ne laissent plus de place à l'hésitation « sur le statut métaphysique du phénomène, sur son identification en transcendance positive ou négative $»^{4}$, mais subordonnent la dimension «verticale» du surnaturel à la logique « horizontale» du réel, brisant du même coup l'alternative fantastique entre les deux mondes. Quant au cadre de la forêt, domestiqué, il ne remplit plus sa fonction « cruciale », ouvrant vers un ailleurs à la fois horizontal et vertical. L'impassibilité du héros à l'égard de son démon persécuteur empêche la «verticalisation de la référence » : la «mise en espace des forces surnaturelles $\rangle^{5}$ se réduit à l'axe horizontal d'une quête héroïque, voire alimentaire ou purement divertissante.

Toutefois, attaché aux valeurs morales, Richard s'emploie à recycler les entreprises de Brundemor, à « blanchir » les êtres et les objets diaboliques, à « convertir en bien tout ce qui vient du monde des enfers $»^{6}$. À son instigation, l'enfant trouvé reçoit de l'archevêque de Rouen le sacrement du mariage chrétien (v.353), tandis que le moine luxurieux, libéré de son péché, devient le plus «preudhomme en la

${ }^{4}$ Fr. Dubost, «Quelque chose que l'on serait tenté d'appeler le fantastique... Remarques sur la naissance du concept », Revue des Langues Romanes, CI, 1997 ${ }^{(2)}$ (Merveilleux et fantastique au Moyen Âge, 2), p. 18.

${ }_{5}$ Id., Aspects fantastiques de la littérature narrative médiévale (XII $\left.{ }^{e}-X I I I^{e}{ }^{s}.\right) . L^{\prime}$ 'Autre, l'Ailleurs, l'Autrefois, Paris / Genève, Champion / Slatkine, 1991 (N.B.M.A., 15), p. 349.

${ }^{6} \mathrm{C}$. Blons-Pierre, «Le monde diabolique dans la version en vers de Richard sans peur $\left(\mathrm{XV}^{\mathrm{e}}\right.$ siècle) », Littérales, 22 (L'épopée tardive. Études réunies et présentées par Fr. Suard), 1998, p. $17-18$. 
«preudhomme en la religion » (v.319). De même, les dons du diable subissent une transmutation: les pommes ne servent plus à un usage personnel, comme ce fut le cas pour Adam et Eve, mais Richard en recueille les pépins et les plante pour les faire fructifier-comme par allusion à la parabole des talents; quant au drap de soie, produit d'importation infernale offert par Hellequin à l'issue de leur entrevue, le duc l'adapte à un usage chrétien en le déposant sur l'autel de sa chapelle, en offrande à Notre Dame (v.199-200). Brundemor lui-même n'échappe pas à cette politique de conversion. Lorsque, au terme de sept tentatives infructueuses, il se présente à Richard sous l'apparence d'un chevalier noir et lui propose ses services de mercenaire, son action devient bénéfique dans la mesure où le duc l'emploie à restaurer l'intégrité de son duché, en repoussant l'envahisseur anglais. L'offensive diabolique est ainsi récupérée au profit de la «légitime défense »? Car le duc se montre soucieux de maintenir l'ordre sur ses terres ${ }^{8}$ : il s'emporte contre les revenants de la «mesnie Hellequin», qui usurpent son droit de chasse (v.97-100). Pour mettre fin aux turbulences des cadavres hantés, il instaure dans tout son duché l'obligation de veiller les morts (v.248-259). Il ira même jusqu'à exporter en enfer l'exercice de sa justice, en allant se battre contre le diable Burgifer, qui a usurpé la sénéchaussée de Brundemor : le duel se déroule sous l'arbitrage du «roy d'enfer » (v. 644) et le démon lésé peut, grâce à la victoire de son champion, goûter à nouveau la pleine jouissance de son fief. Le monde infernal a perdu de son étrangeté : il obéit à la même logique que le monde féodal. En outre, par un renversement significatif, ce n'est pas Richard qui connaît l'« esbahissement» face au surnaturel, mais le diable Burgifer, qui s'étonne du courage et de la vaillance de son adversaire (v.668). Déjà, lors de sa rencontre avec la «mesnie Hellequin », le héros n'avait perdu ni le sens ni la mémoire dans la «carole» magique des revenants. Les épreuves qu'il surmonte avec autant de facilité relèveraient-elles, pour reprendre le jeu de mots d'Eustache Deschamps, d'un " merveillable non merveilleux »", d'un univers qui a perdu son potentiel surnaturel et se réduit à l'exercice d'une faculté jugeante ?

Il n'en reste pas moins qu'à la raison, Richard préfère la force. Des deux modes de «résolution» qu'appelle la «diabolie», ce n'est pas celle des clercs ou des ermites qu'il choisit : il préconise la méthode " héroïque », qui recourt à la prouesse pour éliminer ou maitriser la merveille, tandis que l'explication demeure en suspens. L'auteur se plaît à retarder la révélation finale, les aveux de Brundemor aux enfers, où il expliquera toutes ses tentatives à Richard. Tout au long des séquences fantastiques, le processus de «symbolie » est réduit à l'« ostension » des signes, pur spectacle offert à la jouissance, dans la rétention du sens ${ }^{10}$.

\footnotetext{
${ }^{7}$ Id., Ibid., p. 19.

${ }^{8}$ Cf. E. Gaucher, «Les recettes du diable : le pouvoir et l'argent dans Richard sans Peur », Le prince, l'argent, les hommes au Moyen Âge (Mélanges Jean Kerhervé), Presses universitaires de Rennes, 2008.

${ }^{9}$ E. Deschamps, CEuvres complètes, t.1, Paris, Didot, 1878 (S.A.T.F.), LXXIII, p. 173, v. 10 ; cit. dans C. Ferlampin-Acher, op. cit., p. 45.

${ }^{10}$ J.-R. Valette, «La merveille et son interprétation : l'exemple du Lancelot propre », Revue des Langues Romanes, C, 1996(2) (Merveilleux et fantastique au Moyen Âge), p. 196-197 (sur les modes de résolution) et p.185 (sur le processus de «symbolie »).
} 
Celui-ci n'est donné à Richard qu'à la fin du récit, mais le lecteur sait, depuis le début, que toutes les aventures sont le fait de Brundemor qui a pris congé du " grant maistre d'enfer» (v.34) pour aller tenter sur terre le chevalier sans peur... mais non sans reproche. En effet, le dénouement nous apprend que Richard vit «en peché » (v.728) et que, faute de s'être confessé, il est à la merci des démons ${ }^{11}$. Rien n'est dit sur la nature de ce mystérieux péché, mais il semble permis de l'identifier à l'orgueil, qui engendre une témérité répréhensible, une indifférence excessive à l'égard des dangers dont l'âme est entourée ${ }^{12}$.

Dès lors, le récit peut se lire comme une tentative avortée de «parabole continuée », à la manière de la Queste del Saint Graal ${ }^{13}$. Puisant dans une topique inspirée de la Bible ou dans un registre symbolique bien connu des lecteurs, les épreuves fantastiques invitent à une moralisation: loin de se réduire à des fables divertissantes, elles ont la force de scènes emblématiques et véhiculent une « senefiance» à déceler, sous le voile de la fiction, dans une interprétation allégorique. Mais, à la différence des chevaliers de la Table Ronde qui, inquiets, interrogeaient les ermites sur le sens de leurs aventures, Richard en reste au niveau profane, se contentant d'interroger ses forestiers sur le sexe de l'enfant trouvé ou ses jardiniers sur l'emplacement du pommier disparu. Alors que chaque épisode devrait l'inciter à réviser son comportement, à renier la vanité de ses errances chevaleresques, il lui faut atteindre les profondeurs de l'enfer pour prendre conscience de la « recreantise » à laquelle l'expose ce jeu gratuit avec la peur : à son retour, il réforme son mode de vie par des fondations pieuses et en participant à la croisade aux côtés de Charlemagne. Sa lenteur à gloser les aventures, sa complaisance à prolonger les plaisirs nocturnes (héritage de Robert le Diable, qui consacra ses débuts à de folles errances, dans l'oubli de Dieu) participent du processus parodique, qui semble lié, du moins temporairement, à une « redéfinition profane» du chevalier, à un « retour (...) à une chevalerie séculière $»^{14}$, et donc à un renversement de la hiérarchie fondée sur l'opposition des valeurs terriennes et «célestielles ». D'un point de vue narratologique, cet évidement qui ôte aux «diabolies » leur transcendance répond à la définition du fantastique en tant que merveilleux dont le sens est suspendu sur le mode $\mathrm{du} \mathrm{jeu}^{15}$. Seules les gloses peuvent orienter vers un sens spirituel la lecture d'un roman de chevalerie. Or dans Richard sans Peur, aucun avertissement ne vient interrompre l'errance du héros, jusqu'à sa prise de conscience finale du «péché» qui faisait de lui la proie des démons. Alors, seulement, se dégage un schéma interpréta-

\footnotetext{
${ }^{11}$ On retrouve ici un motif déjà attesté dans la Suite du roman de Merlin (éd. G. Roussineau, 1 vol., Genève, Droz, 2006), §309, 43-46: «Che savons nous que li autres dyables vaurroient tous jours que li pechi de chascun fuissent repost et celé, si que il n'en issist ja de la bouce au pecheour... ».

${ }^{12}$ Sur le manquement de Richard au «don de crainte», voir notre article : «La parodie du fantastique dans Richard sans Peur », art. cit.,en particulier p. 259-260.

${ }^{13}$ Cf. E. Baumgartner, «Achever les aventures dans la Queste del Saint Graal », Méthode!, 2004, p. 11-21.

${ }^{14}$ C. Ferlampin-Acher, op. cit., p. 453.

${ }^{15}$ Id., Ibid., p. 522.
} 
tif qui remet en cause l'excellence du chevalier, à l'instar des leçons qu'adressaient les «preudhommes» aux personnages de la Queste:

La témérité, le désir de tenter l'aventure en même temps que de se laisser tenter par elle, y sont lus en effet comme autant de manifestations d'une confiance en soi condamnable à proportion qu'elle occulte le sentiment d'humilité que devrait susciter, chez l'homme, la conscience de sa condition pécheresse. ${ }^{16}$

En retardant le commentaire édifiant, si discret d'ailleurs que le héros, comme le lecteur, est appelé à deviner le "péché» évoqué, l'auteur permet à la matière romanesque d'évoluer librement, de se poursuivre inlassablement comme une dynamique tournant à vide, sans être assujettie à l'autorité d'un sens unique.

Ainsi, l'accumulation des épreuves diaboliques ne fonctionne plus comme un test de qualification destiné à exalter la prouesse et la sagesse du héros : elle montre au contraire l'indifférence qui frappe la merveille, tant dans son impact affectif que dans son message moral. Richard, par son impassibilité, trahit l'usure des motifs, d'une esthétique et d'une pastorale de la peur que l'habitude a privées d'efficacité. À la différence de Robert le Diable, durement éprouvé par l'affrontement que se livraient en lui Dieu et Satan, le nouveau duc de Normandie semble n'y voir plus qu'un jeu de rôles.

Le motif du mariage accentue encore davantage la différence entre les deux personnages: alors que le père, au terme de sa pénitence, finit ses jours dans un ermitage ou bien, dans les versions tardives de la légende, épouse la fille de l'empereur de Rome pour consolider son alliance avec la Ville Sainte, le fils s'unit à une démone puis, déçu dans ses attentes, fait vœu de ne plus prendre femme, jusqu'à ce qu'un remanieur, dans une mise en prose $\mathrm{du} \mathrm{XVI}^{\mathrm{e}}$ siècle, déroge au motif du «moniage » et célèbre les secondes noces du héros avec une princesse anglaise, où l'on devine l'aboutissement d'une carrière mondaine, validée par les critères courtois $^{17}$.

\section{Le mariage avec le diable}

Dans la version en vers de Richard sans Peur, à laquelle se limite la présente étude, le motif du mariage avec le diable s'inspire à la foi du schéma mélusinien et morganien: Brundemor suit d'abord le trajet de Mélusine en rejoignant Richard dans le monde des mortels puis se «morganise » en l'entraînant en enfer. Mais surtout, l'intention moralisante du récit s'appuie sur la satanisation de la fée, qui s'observe dès le XII ${ }^{\mathrm{e}}$ siècle en corrélation avec le discours de l'Église contre les

\footnotetext{
${ }^{16}$ M. Séguy, Les romans du Graal ou le signe imaginé, Paris, Champion, 2001 (N.B.M.A.., 58), p. 224. On pourrait comparer le «péché » de Richard à celui de Mélyant qui, dans la Queste, pèche non seulement «par excès de confiance en soi, mais aussi par ignorance des codes qui ont cours dans les récits de miracles et les exempla édifiants » (Ibid.).

${ }^{17}$ Voir notre article «Richard sans Peur, du roman en vers au dérimage : merveilles et courtoisie au $\mathrm{XVI}^{\mathrm{e}}$ siècle », Du roman courtois au roman baroque, dir. E. Bury et Fr. Mora, Paris, Les Belles Lettres, 2004, p. 123-134.
} 
superstitions. L'auteur en a peut-être utilisé la récente formulation que lui offrait une légende attestée dans deux textes du $\mathrm{XIV}^{\mathrm{e}}$ et du $\mathrm{XV}^{\mathrm{e}}$ siècle, le Livre de Baudouin de Flandre et le résumé que donne de ce roman Jean d'Outremeuse dans son Myreur des histors ${ }^{18}$. De fait, on relève des ressemblances entre Raimondin, époux de Mélusine, et les deux malmariés que sont Baudouin de Flandre et Richard sans Peur, séduits par une démone.

Tout commence par une situation d'esseulement: le narrateur insiste sur la « disponibilité matrimoniale» et la « singularité sociale» du héros ${ }^{19}$, qui traverse une période de vide affectif et de rupture avec son environnement immédiat. Cet état de détresse atteint son point culminant lors de la rencontre avec la fée : Raimondin vient de tuer accidentellement son oncle et père adoptif, Baudouin s'est attiré l'inimitié de la cour de France en repoussant la main de la fille du roi, et Richard est en manque d'émotions fortes tandis que ses barons lui reprochent de n'avoir point encore pris femme. Tous trois errent, isolés de leur entourage amical, féodal et familial. L'hybris les rend vulnérables, qu'il s'agisse de la douleur qui fait sombrer Raimondin dans la folie ou de l'orgueil qui aveugle Baudouin et Richard. L'inquiétude, l'insomnie, la fatigue voire, pour le duc de Normandie, une «tristour » (v.805) où se lit le désespoir, les prédisposent à devenir la proie d'une puissance merveilleuse ${ }^{20}$. La rencontre et l'union avec la femme surnaturelle semblent inopinément combler l'attente de ces malheureux célibataires, qui croient enfin connaître le bonheur. Mais bien vite, une crise conjugale, le plus souvent liée à la transgression d'un interdit, entraîne la séparation du couple et le départ de la fée, offusquée ou débusquée : perte due, pour Raimondin, à l'indiscrétion qui lui fait découvrir la nature serpentine de Mélusine; pour Baudouin, à l'exorcisme d'un ermite, qui pousse la comtesse de Flandre à confesser son obédience satanique ; et pour Richard, à la malencontreuse tentative d'auto-défense qui lui fait sortir son épée au moment où Brundemor, jouant le scénario de la fausse morte, ressuscite avec fracas. Un regard inquisiteur, un signe apotropaïque, une épée menaçante, voilà ce qui fait fuir une épouse démoniaque. Mais si Mélusine inspire des regrets à celui qu'elle délaisse, conscient des avantages qu'il perd avec elle, il n'en est pas de même dans les deux autres cas, où le héros comprend qu'il a été berné, pris au piège de sa propre vanité, même si, dans Baudouin de Flandre, la démone, «ange déchu [qui] se souvient des cieux »" ${ }^{21}$, aspire plus à se trouver un compagnon d'infortune qu'à tendre, comme dans Richard sans Peur, des pièges dictés par une irréductible perversité. Enfin, notons que la fiction peut elle-même «épouser » l'histoire : les deux créatures diaboliques qu'engendre le conte de Flandre renvoient à des personnages bien réels, Jeanne et Marguerite, filles de Baudouin IX, tandis que le prototype historique de Brundemor, dans la légende de Richard I ${ }^{\text {er }}$ de Normandie, a pu faire l'objet de deux hypothèses :

\footnotetext{
${ }^{18}$ Le Livre de Baudoyn, comte de Flandre, éd. C.-P. Serrure et A. Voisin, Bruxelles, 1836. Sur le traitement de la fée «diabolisée » dans cette légende, voir L. Harf-Lancner, Le Monde des fées dans l'Occident médiéval, Paris, Hachette Littératures, 2003, p. 194-196.

19 J.-J. Vincensini, Pensée mythique et narrations médiévales, Paris, Champion, 1996 (N.B.M.A., 34), p. 152-155.

${ }^{20}$ Cf. C. Ferlampin-Acher, op. cit., p. 108-109.

${ }^{21}$ L. Harf-Lancner, Les Fées au Moyen Âge, Paris, Champion, 1984 (N.B.M.A., 8), p. 403.
} 


\begin{abstract}
l'on peut se demander qui se cache derrière les différentes facettes du personnage de Brundemor : Gunnor, femme réelle de Richard, d'abord sa concubine et de surcroît une incroyante Danoise, autant dire le diable en personne (...)? Mais qui se cache alors derrière Brundemor, libérateur des Anglais? Nous sommes au $\mathrm{XV}^{\mathrm{e}}$ siècle et le personnage de Jeanne d'Arc est dans toutes les mémoires : à la fois figure angélique lorsqu'elle a débarrassé la France des envahisseurs anglais, puis figure démoniaque et hérétique, lorsqu'elle a été brûlée à Rouen... ${ }^{22}$
\end{abstract}

Mais surtout, le mariage diabolique de Richard sert de cadre à la parodie de l'eros courtois. Contrairement à ce qui se passe dans la légende de Baudouin de Flandre, où le signe de la croix et le contact avec les objets sacrés sont appelés à conjurer la présence de la démone, le duc de Normandie n'y recourt pas mais préfère dégainer son épée, geste au demeurant peu courtois à l'égard d'une femme sans défense, comme on le lui reprochera. Or cette arme, interposée entre la dame et le chevalier au cœur d'une nuit peu sûre, ne rappelle-t-elle pas celle qui séparait les corps de Tristan et Iseut endormis dans la forêt du Morrois ${ }^{23}$ ? Le brutal réveil de l'épouse satanisée remplace l'irruption du mari insomniaque et jaloux dont il s'agissait alors de se protéger. Par-delà les différences de contextes, il semble permis d'interpréter le mariage diabolique de Richard comme une réécriture de l' «assag » courtois, même si l'épreuve sert à évaluer non plus la continence du héros, mais son courage. Lorsque, en enfer, Richard prend congé de Brundemor en disant : «ne me tente plus », on peut se demander s'il cherche à fuir la tentation diabolique ou la séduction féminine. La démone fait elle-même référence à la fin 'amor, dans la scène de la fausse mort: prétextant un malaise dû à une soif extrême, elle supplie son époux d'aller lui chercher de l'eau, au nom de la «bonne amour certaine» qu'il lui doit (v.443). Là encore, à travers le motif du « boivre», ressurgit le souvenir de toute une tradition courtoise, de Tristan à Cligès, du vin herbé concocté par la mère d'Iseut et consommé par les amants de Cornouailles, à la «poison » préparée par Thessala pour permettre à Fénice d'échapper aux étreintes de son époux - en d'autres termes, des «noces d'amour et de mort» aux « mirages de l'amour » et à la «mystification de la mort $»^{24}$. Dans Richard sans Peur, le stratagème de la démone consiste à éloigner l'époux naïf pour tuer son chevalier et prendre la fuite. Cela n'empêche pas, à la fin du récit, le diable Burgifer de continuer à filer la métaphore courtoise, en identifiant le combat aux enfers au duel qu'un champion soutient pour sa dame:

«La femme que aviés, Sire duc, espousee,

C'estoit ce grant diable pour qui en celle pree

Contre moy conbatés en bataille ordonnee ». (v.719-721)

${ }^{22}$ C. Blons-Pierre, art. cit., p. 23.

${ }^{23}$ Tristan et Iseut (Béroul), éd. D. Lacroix et Ph. Walter, Paris, Le Livre de Poche, 1989 («Lettres gothiques »), v. 1805-06.

${ }^{24}$ Fr. Gingras, «Les noces illusoires dans le récit médiéval $\left(\mathrm{XII}^{\mathrm{e}}-\mathrm{XIII}{ }^{\mathrm{e}}\right.$ siècles) $)$, Magie et illusion au Moyen Âge (Senefiance, 42), p. 184. 
La réponse de Richard joue sur l'ambiguïté des registres, permise par les métamorphoses successives de Brundemor : celui du «guerredon » due à la démone, conformément au pacte que le héros a contracté avec elle, et celui de la récompense due au chevalier noir, qui a rempli le service d'ost en luttant contre les Anglais :

«Et, pour la courtoisie qu'i [Brundemor] me fist celuy jour,

Pour luy vouldray finer encontre toy l'estour. » (v.743-744)

Dans cette ultime entrevue aux enfers, Brundemor lui-même affiche une étrange gratitude envers Richard, «tout [au] commant» de celui qui le prit pour «femme» (vv.791 et 794)..

L'écriture fantastique transpose donc l'érotique courtoise: le diable et la femme ne maîtrisent-ils pas communément l'art de la déception? La séduction féminine trouve son corollaire dans les tentations dont les puissances succubes entourent les mortels : aussi l'amant «enfantosmé » par Amour et le chevalier persécuté par une démone se rejoignent-ils dans l'expérience d'un jeu pervers, qui les entraîne vers « la grande illusion de la jouissance », " où leur échappe l' " estre » véritable de l'autre qu'ils étreignent $»^{25}$. Tout se passe comme si la rencontre amoureuse ne pouvait se vivre que sous le signe de la faute, d'un péché originel longtemps tenu secret. Depuis la conception du roi Arthur, fruit d'un adultère permis par la magie de Merlin, le sceau de l'illusion diabolique marque les amours des chevaliers arthuriens. De la même manière, la scène primitive de la naissance de Robert le diable, né des œuvres de Satan, semble conditionner, comme une "hypothèque ${ }^{26}$, la vie sentimentale de son fils, Richard. S'agit-il d'agit-il d'automatismes littéraires, ou bien le poids de l'hérédité diabolique, la part de l'indicible, la transgression des tabous, trahissent-ils, dans ces amours romanesques, les peurs latentes liées à la sujétion sexuelle ? Toujours est-il qu'à la fin du Moyen Âge, le renversement parodique introduit un espace de liberté : alors que, dans les romans arthuriens, le motif de la demoiselle prisonnière du diable dans un tombeau traduit les «fantasmes associés à l'imaginaire de l'amour », une « sorte de perversion morbide» qui conduit à penser « la relation amoureuse (...) selon des rapports de dominant à dominé $»^{27}, l^{2}$ « entombement » de la démone et sa fuite dans Richard sans Peur témoignent d'un échec à retenir l'homme dans le mouroir d'un amour illusoire.

La libération du héros s'affirme d'abord par sa décision de ne plus se marier (v.503-506). Si l'attrait féerique resurgit, quelque temps après, à l'annonce d'une chasse au sanglier «faé » « aussi blanc que signe» (v.597), le projet reste sans lendemain. Richard ne peut renouer avec les plaisirs de la chasse car l'enfer l'appelle. Là, par une ultime victoire, il s'affranchit définitivement de l'emprise diabolique. Dès lors, il peut mener « saincte vie » et mettre sa vaillance au service de la croisade

${ }^{25}$ Id., ibid., p. 180.

${ }^{26}$ Id., ibid.

${ }^{27}$ Fr. Dubost, op. cit., p. 416-417 (à propos de l'Âtre périlleux et des Continuations du Conte $d u$ Graal). 
(v.808-810). Le mariage diabolique apparaît donc comme une felix culpa, qui aide le héros à prendre conscience de sa « recreantise», à repousser les chimères du diable et à retourner à la cour pour y défendre la bonne cause. Le dénouement proclame la fin des illusions et le retour au devoir vassalique. L'auteur inverse ici les données du Livre de Baudouin de Flandre, où le héros, coupable de sacrilège pour avoir rompu son alliance avec la monarchie capétienne en refusant la main de la fille du roi, doit endurer jusqu'à la mort le châtiment que Dieu lui envoie par l'intermédiaire de l'épouse diabolique et de ses filles perverses : le fantastique sert alors à transmettre une leçon politique sur le soutien indéfectible que tout seigneur doit à son roi et à justifier, par le manquement à ce principe, « la décadence du lignage des comtes de Flandre » et la perte de leurs possessions territoriales ${ }^{28}$.

Mais si le duc de Normandie peut retourner à ses activités guerrières, le romancier ne s'y attarde pas, à l'inverse des chroniqueurs ${ }^{29}$ attachés à glorifier le souvenir de Richard I ${ }^{\text {er }}$. L'enjeu diverge, d'un genre à l'autre. Là où l'histoire privilégie les exploits militaires, le roman se nourrit de la quête d'aventures ${ }^{30}$. La fiction prend fin quand disparait le désir qui l'a fait naître. Et, le diable, en tant que moteur de l'action, apparaît comme un double de l'auteur, maître d'une diégèse qui soumet à l'épreuve de l'illusion non seulement la volonté du héros mais aussi celle du lecteur.

\section{Jouer à se faire peur}

Si l'impassibilité de Richard permet de faire durer les aventures, elle demande aussi à être interrogée, sur le plan idéologique. Le personnage, que les démons eux-mêmes qualifient de pécheur, ne semble pas illustrer les affirmations de Jean Delumeau, pour qui l'essor de la bourgeoisie et la perte de confiance dans l'élite aristocratique auraient contribué, au $\mathrm{XV}^{\mathrm{e}}$ siècle, à renforcer, par réaction, «l'exaltation sans nuance de la témérité» dans la littérature épique et narrative ${ }^{31}$. Richard s'apparente plutôt aux chevaliers orgueilleux des chansons de geste, qui, tel Mabrien, poussent leur «anti-peur» «à rechercher avec bonheur et enthousiasme toutes les situations de danger $\aleph^{32}$. Depuis l'exemple fourni par Roland, on sait les reproches que s'attire «l'inconscience héroïque $»^{33}$ : la démesure semble bien être le péché capital d'une chevalerie surdimensionnée, oublieuse de son humanité et, par là même, de sa vulnérabilité face à la réalité peccamineuse. L'absence de peur est liée à l'oubli de Dieu, «qui donne la ligne de partage entre le bien et le mal » et de la

${ }^{28}$ C. Gaullier-Bougassas, «Temps historique et temps romanesque : Saladin et Baudouin de Flandre », Dire et penser le temps au Moyen Âge, études recueillies par E. Baumgartner et L. Harf-Lancner, Paris, Presses de la Sorbonne Nouvelle, 2005, p. 231.

${ }^{29}$ Dudon de Saint-Quentin, Guillaume de Jumièges puis Wace et Benoît de Sainte-Maure.

${ }^{30}$ Sur les différents textes qui évoquent le personnage historique de Richard, voir N. Cazauran, «Richard sans peur : un personnage en quête d'auteur », Travaux de littérature, IV, 1991, p.21-43.

${ }^{31}$ J. Delumeau, La Peur en Occident, XIV ${ }^{\mathrm{e}}$ - XVIII ${ }^{\mathrm{e}}$ siècles, Paris, Fayard, 1978, p. 3.

${ }^{32}$ B. Ribémont, «La Peur épique », à paraître dans le Moyen Âge.

${ }^{33}$ J. Flori, «Le héros épique et sa peur (du Couronnement de Louis à Aliscans) », PRIS-MA, t.X, nº1, janvier-juin, 1994, p. 27. 
croisade, comme le déplore le pape Pie II qui, en 1459, au Congrès de Mantoue, accuse les chrétiens de son temps d'être endormis dans un «profond sommeil», en «serviteurs inutiles $»^{34}$. En d'autres termes, exorciser la peur, n'est-ce pas libérer l'espace du rêve et du jeu?

Par un renversement burlesque de l'idéologie courtoise, où le chevalier devait prouver sa vaillance et sa témérité aux yeux de sa dame, Richard doit avoir peur pour mériter les faveurs de sa démone. Or ce sentiment tardant à se manifester, le désir reste entier, et avec lui la mécanique répétitive du jeu : la logique ressemble bien à celle de la fin'amor, qui se nourrit du manque et s'éteint dans la satisfaction du plaisir consommé. Le duc de Normandie se livrerait-il, comme bien d'autres nobles de la fin du Moyen Âge, à une mise en scène qui lui permette de s'illustrer sans contrainte dans un monde fictif, artificiel, à l'instar des héros arthuriens ? À ceci près qu'il se désolidarise des enjeux métaphysiques, de cette sacralisation de l'amour auxquels étaient soumis ses modèles, quêteurs du Graal ou de la dame divinisée : à Richard l'impassible ne reste plus que le divertissement, une bien pâle imitation de la geste des chevaliers du temps jadis, vidée de toute transcendance et bien vite corrodée par l'ennui.

Bien plus, par une sorte de mise en abyme, le jeu avec le diable reflète le plaisir du texte. On a pu dire que l'aristocratie du $X^{\mathrm{e}}$ siècle se livrait à une consommation bovaryste de la littérature chevaleresque ou à un don Quichottisme avant la lettre, en calquant son existence sur les romans arthuriens, notamment dans les joutes et pas d'armes, où les nobles portaient les noms et les couleurs des héros de la Table Ronde. Or le comportement de Richard sans Peur n'est guère différent du mimétisme de ces lecteurs trop enclins à briser les frontières du réel et de l'imaginaire. Il consiste, sinon à s'identifier, tel Narcisse, du moins à se consacrer aux reflets flatteurs que lui tend le miroir de la fiction. Au cours de ses nocturnes diaboliques, Richard se laisse entraîner dans une sorte de « visite guidée livresque » ou de «pèlerinage» «sur les sites de la mémoire arthurienne». Nous avons affaire à un roman qui, par le constant recours à l'intertextualité, relève de «l'esthétique du médaillon », du « livre-reliquaire» donnant à voir « une somme du matériau littéraire », à qui il rend hommage mais dont il gauchit cependant les enjeux par le détournement parodique, telle une satura lanx $x^{35}$.

Le texte parodique relève bien de l'opération dia-bolique, qui « consiste à séparer ce qui doit être réuni $»^{36}$. Quand Brundemor, sous l'apparence du roi Hellequin, prédit à Richard qu'aucun diable ne lui fera jamais de mal (v.155), il sape son propre travail, en démonte les mécanismes, comme par auto-dérision. Paradoxalement, les démons jouent double jeu, en fournissant à la fois l'aventure et la glose, le fantasti-

${ }^{34}$ V. Guyen-Croquez, «Peur et courage dans les Croniques et conquestes de Charlemaine de David Aubert, texte du XV ${ }^{\mathrm{e}}$ s. », Médiévales, 6, 2004, p. 87.

${ }^{35}$ Nous empruntons ces expressions à Patricia Victorin (Ysaïe le Triste Une esthétique de la confluence, Paris, Champion, 2002, p. 342, 382-383 et 455-456. Cf. aussi Id., «Entre balbutiement et radotage : enfance, répétition et parodie dans le roman arthurien du Moyen Âge tardif », Études françaises, $42^{(1)}$ (De l'usage de vieux romans), 2006, p.63-89.

${ }^{36} \mathrm{Cl}$. Reichler, La diabolie. La séduction, la renardie, l'écriture, Paris, Éditions de minuit, 1979, p. 11. 
que et l'exégèse moralisatrice. Tel Merlin, cet Antéchrist racheté par le baptême, qui, dans la Suite Huth, éclaire Arthur sur le sens de sa vie, Brundemor et Burgifer portent un regard omniscient sur celle de Richard (v.725-726). La scène finale donne lieu à «jugement dernier» parodique, où la morale s'énonce par le truchement de l'instance démoniaque : Richard se voit accusé d'être un pécheur non repenti (v.729730), et, par là même, de s'être laissé prendre aux pièges de la « diabolie ».

La sentence, bien sûr, témoigne d'un orgueil luciférien, car Richard, tout au long de ses épreuves, a su « exorciser» le mal en lui ôtant sa transcendance. Adepte de la « renardie», il a « invers[é] l'espace théologique vers le bas », c'est-à-dire vers les besoins du corps, les vanités terrestres. En s'offrant comme « une victime, mais active, une dupe, mais consentante $»^{37}$ des démons, il a joué avec son propre désir, s'est laissé abuser sans se porter solidaire dans la mise en scène de ses propres fantasmes - de ses propres démons.

Ainsi, le roman de Richard sans Peur s'inscrit dans une filiation littéraire, qu'il ne cesse de montrer du doigt tout en sortant de la voie qu'elle a tracée. Au rire et à la prescience du diable, se substituent ceux du lecteur, qui jouit du plaisir de la reconnaissance, sur le sentier des lieux communs, et de l'effet de surprise, dans les écarts parodiques.

Mais le traitement parodique des tentations diaboliques ne doit pas être interprété en termes de reniement ou de "dénonciation d'invraisemblance». En cette fin du Moyen Âge où prolifèrent les représentations du diable, si l'on peut dire que le merveilleux est avant tout un jeu, c'est parce qu'il évacue « des croyances auxquelles une adhésion ferme serait supposée » et repose, au contraire, "sur la mise entre parenthèses provisoires du dogme chrétien rassurant pour jouer avec la peur et le plaisir $\aleph^{38}$. Le fantastique se réduit à un procédé littéraire.

Et si Richard sans Peur est le fils de Robert le Diable, on peut aussi lui trouver d'autres liens de parenté. En effet, noctambule, insomniaque à ses heures, il affiche une agitation nocturne qui évoque le somnambulisme d'un Pierre de Béarn, dont Froissart nous raconte qu'il se levait la nuit et s'armait pour combattre un ennemi imaginaire ${ }^{39}$. Or un tel comportement, au Moyen Âge, avait partie liée à la folie $^{40}$. Richard est possédé, non pas tant par le démon mais plutôt par son goût pour les aventures chevaleresques. Il ressemble aussi à Gaston Phébus qui, malgré son surnom solaire, menait une vie nocturne et à qui Froissart lisait chaque nuit quelques pages du Méliador: or le premier personnage qui apparait dans ce roman, Camels de Camois, a peur de dormir seul mais ne craint pas de chevaucher la nuit dans la forêt solitaire : ce somnambule, dominé par la puissance maléfique d'Hécate, déesse des

\footnotetext{
${ }^{37}$ Id., Ibid.,p. 97-98 et 214.

${ }^{38}$ C. Ferlampin-Acher, «Merveilleux et comique dans les romans arthuriens français (XII ${ }^{\mathrm{e}}$ $\mathrm{XV}^{\mathrm{e}}$ siècles) ", Arthurian literature, t.XIX, 2003, p. 36 et 46-47.

${ }^{39}$ Chroniques, III, 14 (éd. Kervyn de Lettenhove, t.XI, p. 100-106). Cf. M. Zink, «Froissart et la nuit du chasseur », Poétique, 11, 1980, p. 60-77.

${ }^{40}$ J.-M. Fritz, Le discours du fou au Moyen Âge, Paris, P.U.F., 1992 («Perspectives littéraires »), p. 350.
} 
visions nocturnes, la défie victorieusement à l'état de veille par ses chevauchées nocturnes. Le duc de Normandie reproduit, sur le mode parodique, le souvenir d'ancêtres littéraires.

Élisabeth Gaucher Université de Nantes 